\title{
Atividade antimicrobiana e rendimento do óleo essencial de Pimenta pseudocaryophyllus var. pseudocaryophyllus (Gomes) Landrum, Myrtaceae
}

\author{
YOKOMIZO, N.K.S'.; NAKAOKA-SAKITA, M."* \\ 1'Instituto Florestal - Secretaria do Meio Ambiente, Seção de Fitotecnia Parasitológica e Laboratório de Fitoquímica, \\ Caixa Postal 1322, 02377-000 São Paulo (SP).E-mail: massako_nakaoka@yahoo.com.br
}

\begin{abstract}
RESUMO: Com o objetivo de verificar o potencial antimicrobiano de Pimenta pseudocaryophyllus var. pseudocaryophyllus, foi avaliado em condições de laboratório o óleo essencial extraído de suas folhas quanto à atividade nos microrganismos: Aspergillus niger, Penicillium verrucosum, Escherichia coli, e Staphylococcus aureus. A metodologia utilizada foi o método do papel de filtro embebido no óleo essencial a ser testado. A extração do óleo essencial foi feita por hidrodestilação em aparelho Clevenger modificado por Wasicky em 1963. Testou-se o óleo essencial de plantas provenientes de Campos do Jordão e de Itaquaquecetuba. Os resultados obtidos mostraram que o óleo da planta procedente de Campos do Jordão apresentou atividade antimicrobiana muito próxima à do citral, inibindo em $72,7 \%$ e $71,5 \%$, respectivamente, o crescimento dos microrganismos; porém, foi significativamente superior ao da planta procedente de Itaquaquecetuba, que apresentou $38,8 \%$ de inibição. Quanto aos microrganismos, a inibição do crescimento foi maior para P. verrucosum $(83,3 \%)$, seguido por A. niger $(71,9 \%)$ e S. aureus $(70,3 \%)$, que não apresentaram diferença entre si. A menor inibição foi apresentada por E. coli $(18,4 \%)$. Com exceção de E. coli, que apresentou a mesma característica de inibição para os dois óleos e o citral, todos os demais fatores apresentaram interação significativa, demonstrando especificidade de reação para cada um dos níveis desses fatores e o potencial antimicrobiano, do óleo essencial de Pimenta pseudocaryophyllus var. pseudocaryophyllus. O rendimento do óleo essencial da planta coletada em Campos do Jordão foi de $2,3 \%$ e da coletada em Itaquaquecetuba foi de $1,9 \%(\mathrm{p} / \mathrm{v})$.
\end{abstract}

Palavras chave: Pimenta pseudocaryophyllus, óleo essencial, rendimento, atividade antimicrobiana.

ABSTRACT: Antimicrobial activity and essential oils yield of Pimenta pseudocaryophyllus var. pseudocaryophyllus (Gomes) Landrum, Myrtaceae. The essential oils of Pimenta pseudocaryophyllus var. pseudocaryophyllus specimens from two distinct locations: Campos do Jordão and Itaquaquecetuba, were tested to detect antimicrobial activity to the microorganisms Aspergillus niger, Penicillium verrucosum, Escherichia coli and Staphylococcus aureus in medium culture. The method utilized was impregnated filter paper in essential oils. The essential oil extraction was made through method of Clevenger modified for Wasicky in 1963, for the hydrodistilation. The monoterpene citral was tested under the same conditions of the essential oils. The microorganisms development under these conditions presented the following results: the oil of P. pseudocaryophyllus var. pseudocaryophyllus from Campos do Jordão had the same antimicrobial activity that citral, inhibiting in $72,7 \%$ and $71,5 \%$, the growth of the microorganisms, significantly higher than the level of the oil originated from Itaquaquecetuba that presented $38,8 \%$ of inhibition. About to the microorganisms, the growth inhibition was higher for P. verrucosum $(83,3 \%)$, followed by A. niger $(71,9 \%)$ and S. aureus $(70,3 \%)$. The minimal inhibitory activity was presented by E. coli (18,4\%). Except for E. coli, which presented the same characteristic of inhibition for two oils and the citral, all the other factors presented significant interaction, showing specificity of reaction for each one of the levels of these factors. These data demonstrated the antimicrobial potential of the essential oil of the Pimenta pseudocaryophyllus var. pseudocaryophyllus The yield of essential oil collected in Campos do Jordão was 2,3\% and the species collected in Itaquaquecetuba was 1,9\% (w/v).

Keywords: Pimenta pseudocaryophyllus, essential oil, yield, microbial activity.

Recebido para publicação em 09/08/2012

Aceito para publicação em 12/03/2014

10.1590/1983-084X/12_097

Rev. Bras. PI. Med., Campinas, v.16, n.3, p.513-520, 2014. 


\section{INTRODUÇÃO}

A produção de óleos essenciais no Brasil é destacada por Bizzo et al. (2009), que, ao lado da Índia, China e Indonésia são considerados os 4 grandes produtores mundiais. No período de 2005 a 2008, a exportação de óleos essenciais de cítricos pelo Brasil foi de 287.799 t. Em 2004, as exportações do Brasil em óleos essenciais registraram valor recorde histórico de US\$98 milhões, revelando-o como $4^{\circ}$ maior exportador de óleos essenciais do mundo (Barata et al., 2005).

Os óleos essenciais constituem um grupo variável e complexo, com necessidade de se compreender e conhecer os mecanismos que regulam a sua produção e sua expressão na natureza. São substâncias integrantes das engrenagens que constituem os ecossistemas e estudos sobre esses óleos essenciais poderão aprofundar o conhecimento da biodiversidade odorífera brasileira e contribuir para o bem estar e a saúde do povo (Gottlieb \& Borin, 2003).

As substâncias voláteis produzidas pelo metabolismo secundário, os fenilpropanóides e, especialmente, os terpenóides são os principais constituintes dos óleos essenciais. Grande parte dos fenilpropanóides são voláteis com ação biológica e é extraído, com arraste a vapor, substâncias com baixo peso molecular (Craveiro \& Machado, 1986).

A família Myrtaceae, apresenta espécies com interesse medicinal, e, em destaque, o gênero Pimenta (Paula et al., 2010)

Pimenta pseudocaryophyllus var. pseudocaryophyllus (Gomes) Landrum, é uma espécie arbórea conhecida como chá da terra, louro, louro da terra. As folhas desta espécie são utilizadas popularmente na forma de chá de efeito calmante. Apresenta aroma semelhante ao da erva cidreira. A família Myrtaceae compreende cerca de 4000 espécies (Landrum \& Kawazaki, 1997), com distribuições tropicais e subtropicais, e compreende cerca de 130 gêneros (Barroso et al., 1984).

Inúmeras espécies da família Myrtaceae são produtores de óleo essencial e apresentam atividade biológica como Myrtus communis var. italica estudada por Gauthier et al. (1989), que detectaram cineol, $\alpha$-pineno e linalol como principais componentes, e que apresentam atividade contra Pediculus humanus capitis.

No estudo da composição química e testes de atividade antifúngica do óleo essencial das folhas de Pimenta pseudocaryophyllus, coletada na região de Telêmaco Borba, Estado do Paraná, Custódio et al. (2007) obtiveram rendimento de 1,1\% pelo processo de hidrodestilação em aparelho Clevenger. O óleo apresentou atividade antifúngica contra Alternaria sp., Botryosphaeria ribis, Botryosphaeria sp. e Aspergillus niger. Os autores encontraram o eugenol como principal constituinte do óleo essencial, substância com comprovada ação antifúngica.

Quanto à composição química do óleo essencial de Pimenta pseudocaryophyllus var. pseudocaryophyllus, procedente de Campos do Jordão, Nakaoka Sakita et al. (1994) encontraram como principais componentes o neral $(27,847 \%)$ e o geranial $(34,256 \%)$ isômeros conhecido como citral. O citral é amplamente utilizado na fabricação de perfumes e de refrigerantes. Além deste isômero foi encontrado também linalol $(5,178 \%)$, geraniol $(4,82 \%)$, $\alpha$-pineno $(1,35 \%), \beta$-pineno $(2,67 \%)$, terpinen-4-ol $(0,308 \%)$, terpinoleno $(0,308 \%), 1,8$ cineol $(0,104 \%)$, limoneno $(0,150 \%)$, $\alpha$-terpineol $(0,488)$.

Estudando o óleo essencial de Pimenta pseudocaryophyllus coletadas na llha do Cardoso e em Paranapiacaba, (Lima et al., 2006) encontraram resultados semelhantes na atividade antimicrobiana contra Pseudomonas aeruginosa e Staphylococcus aureus. Quanto a Candida albicans, o melhor resultado foi à espécie coletada em Paranapiacaba. O principal componente do óleo essencial da espécie coletada na Ilha do Cardoso foi o eugenol enquanto que a espécie de Paranapiacaba apresentou como principal componente o 4-metil-eugenol.

Utilizando extratos etanólicos brutos das folhas de P. pseudocaryophyllus, coletadas em Brasília, DF e São Gonçalo do Abaeté (MG), (Paula et al., 2009) verificaram atividade antimicrobiana contra bactérias Gram positivas Staphylococcus aureus, Micrococcus luteus, M. roseus, Bacillus cereus, B. atrophaeus, B. stearothermophilus e frente as Gram negativas Pseudomonas aeruginosa, $P$. stutzeri e frente a levedura Candida albicans. Compostos fenólicos, taninos, flavonóides e óleos essenciais foram identificados na planta e podem estar envolvidos na ação antimicrobiana.

A atividade antibacteriana e antifúngica de Pimenta racemosa var. racemosa P.Miller, Myrtaceae, foram verificadas por Aurore et al.(1998) que determinaram a concentração inibitória mínima e máxima utilizando método de diluição em meio sólido. Detectaram no óleo essencial de $P$. racemosa a presença de neral $(31,7 \%)$ e geranial $(40,3 \%)$, com atividade de inibição contra Mycobacterium smegmatis, Candida albicans, Escherichia coli, Staphylococcus aureus e Enterococcus faecium.

Quanto à atividade biológica de citral (geranial - 45,9\% e neral - 33,5\%) obtido, principalmente, de Cymbopogon citratus (DC) Stapf, Chalchat et al. (1997) encontraram uma elevada ação inibitória em Staphylococcus aureus e Escherichia coli. Extraído de Cymbopogon citratus. Pereira et al. (2004) verificaram a inibição de 89,9\% 
para bactéria Escherichia coli, 85,7\% Klebisiella pneumoniae, 16,6\% para Proteus mirabilis, porém, nenhuma atividade contra Klebsiella oxytoca e Pseudomonas aeruginosa.

Dentre as espécies estudadas por Apel et al. (1998), M. eugeniopsoides apresentou maior atividade frente a todos os microrganismos testados e $M$. cucullata, inibição total contra $S$. cereviseae. Segundo os autores, as principais substâncias encontradas nas espécies estudadas, com reconhecida ação antimicrobiana, foram: terpinen-4-ol, terpinoleno, I,8-cineol, linalol e $\alpha$-terpineol.

A composição química e atividade antimicrobiana do óleo essencial de Marlierea eugeniopsoides e M. obscura (Myrtaceae), foram efetuadas por Limberger et al. (1998) pelo método de difusão em agar, verificando excelente atividade inibitória contra Staphylococcus epidermidis, S. aureus, Micrococcus luteus e Sacharomyces cerevisiae.

O estudo da composição química do óleo essencial e a atividade antimicrobiana de Eugenia speciosa CAMB. (Myrtaceae) contra bactérias Staphylococcus aureus e Echerichia coli, Viane et al. (1998) usando o método de difusão em agar verificaram inibição, principalmente, a $S$. aureus identificando os compostos: limoneno $(33,7 \%)$, terpineol (18,9\%), e $\alpha$-pineno $(5,8 \%)$.

Considerando o potencial da espécie, o interesse, e a utilização cada vez maior da população por produtos naturais, este trabalho teve como objetivo verificar a atividade antimicrobiana do óleo essencial de Pimenta pseudocaryophyllus var. pseudocaryophyllus (Gomes) Landrum.

\section{MATERIAL E MÉTODOS}

\section{Coleta e identificação botânica}

Amostras de Pimenta pseudocaryophyllus var. pseudocaryophyllus (Gomes) Landrum foram coletadas nas seguintes localidades:

a) Município de Campos do Jordão, Estado de São Paulo, SP., coordenadas 22\%44'19" S e 4535'31" W, especificamente no Parque Estadual de Campos do Jordão, altitude aproximada de $1.808 \mathrm{~m}$, em relevo de colinas e morros altos (Ross \& Moroz, 1997). Segundo Köppen, o clima é subtropical de altitude, mesotérmico úmido sem estiagem, com temperatura média anual mínima de $14,3^{\circ} \mathrm{C}$ e máxima de $15,3^{\circ} \mathrm{C}$. Exsicatas do material encontram-se depositadas no Herbário $D$ Bento Pickel sob o registro $n^{\circ}$ 13083

b) Município de Itaquaquecetuba, Estado de São
Paulo, SP., coordenadas Lat. sul: $23^{\circ} 30$ e longitude: W.Gr. $46^{\circ} 22$, cujas amostras foram coletadas em um único indivíduo, em altitude aproximada de $764 \mathrm{~m}$, segundo a classificação em relevo de Ross \& Moroz (1997) em região morros baixos. O clima, segundo Köppen, é caracterizado pelo clima tropical de altitude, com chuvas no verão e seca no inverno, com a temperatura média do mês mais quente superior a $22^{\circ} \mathrm{C}$. Exsicatas do material encontram-se depositadas no Herbário $D$ Bento Pickel sob o registro $n^{\circ}$ 33.370 .

\section{Obtenção dos óleos essenciais}

O material coletado foi colocado em local ventilado à temperatura ambiente para evitar a fermentação. Foi seco e estabilizado à temperatura de $40^{\circ} \mathrm{C}$ em estufa com circulação de ar por 24 horas. O material estabilizado foi pulverizado em um moinho de faca e martelo.

Para destilação do óleo essencial, utilizouse $400 \mathrm{~g}$ de folhas secas e pulverizadas. A extração foi efetuada pelo método de CLEVENGER, modificado por Wasicky (1963), pela hidrodestilação, por um período de 3 (três) horas.

A título de comparação foi utilizado citral, como tratamento padrão.

\section{Microrganismos}

Duas bactérias e dois fungos, abaixo caracterizados, foram utilizados no presente ensaio. -Escherichia coli, da coleção do Instituto Biológico, SP;

- Staphylococcus aureus, da coleção do Instituto Biológico, SP;

-Aspergillus niger, da coleção de fungos do Instituto de Botânica, SP e

- Penicillium verrucosum, exemplar de número 1712, da coleção de fungos do Instituto de Botânica, SP.

\section{Atividade Antimicrobiana}

A atividade antimicrobiana foi realizada através da utilização do método do papel embebido com o óleo. Papel de filtro CAAL, $80 \mathrm{~g}$, foi recortado em discos com $1 \mathrm{~cm}$ de diâmetro e esterilizados em estufa a $150^{\circ} \mathrm{C}$ por 60 minutos e embebidos no óleo essencial a ser testado. Para óleos essenciais mais viscosos, foi utilizado álcool etílico para dissolver e obter uma melhor impregnação dos discos de papel de filtro.

Como controle absoluto, o disco de papel de filtro apenas autoclavado, sem nenhuma impregnação. Utilizando a metodologia do Plant Pathologist's Pocketbook (1968) as Placas de Petri 
contendo Agar Nutriente e Agar Sabouraud Dextrose foi inoculado com uma suspensão de bactérias e fungos respectivamente.

A suspensão foi obtida através da introdução de uma solução de água destilada esterilizada e Tween a 0,1\% em tubos com colônias de dois dias das bactérias e de 5 dias dos fungos.

Após agitação manual, a suspensão obtida foi diluída em $50 \mathrm{~mL}$ água destilada esterilizada e 0,2 $\mathrm{mL}$ da suspensão resultante foram transferidos para as placas de Petri e espalhados de maneira uniforme na superfície do meio. Através do método da diluição em série, seguido de plaqueamento, estimou-se que cada placa de Petri foi inoculada com:

-227000 esporos de Staphylococcus aureus,

-421500 esporos de Escherichia coli, -56870 propágulos de Aspergillus niger, e verrucosum.

-317500 propágulos de Penicillium

Um disco de papel de filtro impregnado com o óleo essencial foi depositado no centro de cada placa. Placas inoculadas com bactérias foram incubadas em estufa a $34^{\circ} \mathrm{C}$ por 48 horas e placas com fungos a $25^{\circ} \mathrm{C}$ por 70 horas. Após este período foi realizada a avaliação dos efeitos produzidos pelos óleos essenciais no crescimento dos microrganismos, medindo-se o halo de inibição em torno do papel de filtro.

A leitura em centímetros foi transformada em Porcentagem de Inibição do Crescimento PIC, conforme metodologia de Edington \& Khew (1971). Para fins de processamento estatístico esses dados em PIC foram transformados em asen $J(X+00,1 / 100)$, utilizando o programa ESTAT - Sistema para Análises Estatísticas (V. 2.0), da UNESP, Campus de Jaboticabal.

\section{RESULTADOS E DISCUSSÃO}

\section{Rendimento}

A extração do óleo essencial de folhas de Pimenta pseudocaryophyllus var. pseudocaryophyllus apresentou rendimento da ordem de 2,3\% e 1,9\% $(p / v)$, para as procedências de Campos do Jordão e Itaquaquecetuba, respectivamente. Pode ser considerado um rendimento bom, conforme consta na (Tabela 1).

Este elevado rendimento em óleos essenciais torna a Pimenta pseudocaryophyllus var. pseudocaryophyllus uma espécie interessante

Tabela 1. Comparação do rendimento de óleos essenciais de outras espécies e respectivos autores

\begin{tabular}{llc}
\hline Espécie & Autor & $\begin{array}{c}\text { Rendimento } \\
\text { Peso/volume }\end{array}$ \\
\hline $\begin{array}{l}\text { P. pseudocaryophyllus var. pseudocaryophyllus } \\
\text { procedente de Campos do Jordão }\end{array}$ & Neste experimento & $2,3 \%$ \\
\hline $\begin{array}{l}\text { P. pseudocaryophyllus var. pseudocaryophyllus } \\
\text { procedente de Itaquaquecetuba }\end{array}$ & Neste experimento & $1,9 \%$ \\
\hline P. pseudocaryophyllus & & $1,0 \%$ \\
\hline Pseudocaryophyllus pabstianus Legrand. & Custodio et al. (2010) & $0,8 \%$ a 1,2\% \\
\hline Pseudocaryophyllus jaccoudii Mattos & Campos Correa \& Gotllieb (1970) & $2 \%$ \\
\hline Pseudocaryophyllus guilli (Speg.). Burr. & Jardim \& Jaccoud (1965) & 0,17 a 0,21\% \\
\hline Eugenia dysinterica & Fenik \& Retamar (1972) & $0,15 \%$, \\
\hline Myrcia acuminatissima & Paula et al. 1998 & $0,12 \%$ \\
\hline Myrcia bombicina & Henriques et al. (1997) & $0,95 \%$ \\
\hline Myrcia fallax & Henriques et al. (1997) & $0,35 \%$ \\
\hline Myrcia glaba & Henriques et al. (1997) & $0,11 \%$ \\
\hline Myrcia multiflora & Henriques et al. (1997) & $0,20 \%$ \\
\hline Myrcia polyantha D. C. var. coriacea Bg & Henriques et al. (1997) & $2 \%$ \\
\hline Calyptranthes spruceana Berg & De Alencar et al. (1971) & $1,7 \%$ \\
\hline Calyptranthes sp. & Campos Corrêa et al (1972) & $2 \%$ \\
\hline
\end{tabular}

Rev. Bras. PI. Med., Campinas, v.16, n.3, p.513-520, 2014. 
e promissora, não só quanto ao rendimento, como também pelos seus principais constituintes químicos, havendo necessidade de mais estudos, inclusive filogenéticos, envolvendo outras procedências, e mais variáveis, para explicar a variabilidade no rendimento do óleo essencial.

Em trabalho anterior, Nakaoka-Sakita et al. (1994) encontraram no óleo essencial das folhas de P. pseudocaryophyllus var. pseudocaryophyllus neral $(27,847 \%)$ e geranial $(34,256 \%)$ isômeros conhecido como citral. O citral é amplamente utilizado na fabricação de perfumes e de refrigerantes. Além deste isômero foi encontrado também linalol $(5,178 \%)$, geraniol $(4,82 \%), \alpha$-pineno $(1,35 \%)$, $\beta$-pineno $(2,67 \%)$, terpinen- 4 -ol $(0,308 \%)$, terpinoleno $(0,308 \%), 1,8$ cineol $(0,104 \%)$, limoneno $(0,150 \%), \alpha$-terpineol $(0,488)$.

Na Pimenta pseudocaryophyllus coletadas em Paranapiacaba Lima et al. (2006) encontraram 4-metil-eugenol e eugenol na espécie coletada na Ilha do Cardoso. Os autores encontraram melhores resultados contra Escherichia colli, Pseudomonas aeruginosa, e Staphyloccoccus aureus, com a espécie coletada na llha do Cardoso. As espécies coletadas de ambas as localidades apresentaram resultados similares. Entretanto, contra Candida albicans, o melhor resultado foi com a espécie coletada em Paranapiacaba.

Verificou-se que tanto Lima et al. (2006) como Custódio et al. (2007), que trabalharam com a Pimenta pseudocaryophyllus encontraram eugenol e 4-metil-eugenol como constituinte principal do óleo essencial.

Quanto à atividade biológica do citral presente no óleo essencial de Pimenta pseudocaryophyllus var. pseudocaryophyllus, os resultados encontrados, são concordantes com os trabalhos efetuados por Aurore et al. (1998), com Pimenta racemosa, Myrtaceae, e de Chalchat et al. (1997), com Cymbopogon citratus, ambas espécies contendo neral e geranial (citral) como principais componentes com atividade inibitória contra Escherichia coli e Staphylococus aureus.

Os mesmos microorganismos foram utilizados no presente trabalho verificando que a atividade do óleo coletado em Campos do Jordão foi igual ao óleo padrão de citral utilizado. A espécie coletada em Campos do Jordão apresentou maior atividade em comparação à espécie coletada em Itaquaquecetuba.

A atividade antimicrobiana de terpinen-4-ol, terpinoleno, 1,8-cineol, linalol, limoneno, $\alpha$-pineno e $\alpha$-terpineol são encontradas em inúmeras espécies da família Myrtaceae contra Staphylococcus aureus e Echerichia coli Apel et al. (1998), Limberger et al. (1998) e Viane et al. (1998). Os mesmos componentes também foram encontrados por Nakaoka Sakita et al. (1994) na Pimenta pseudocaryophyllus var. pseudocaryophyllus.

Estes componentes, presentes no óleo essencial de $P$. pseudocaryophyllus var. pseudocaryophyllus poderão ter atuado juntamente com o citral na atividade inibitória dos microorganismos utilizados no presente trabalho. A (Tabela 2) apresenta os dados médios, em porcentagem de inibição de crescimento das bactérias e fungos submetidos a crescimento em meio de cultura contendo óleo essencial de Pimenta pseudocaryophyllus var. pseudocaryophyllus. Observam-se diferentes padrões de desenvolvimento dos microrganismos, cuja diferença é comprovada pela análise de variância apresentada.

Observa-se que entre os microrganismos, os fungos apresentaram maior inibição aos óleos essenciais testados, na seguinte seqüência: Penicillium verrucosum apresentou o maior PIC (Porcentagem de Inibição de Crescimento); Aspergillus niger, que não foi significativamente diferente da bactéria Staphylococcus aureus, e Escherichia coli, com menor PIC.

Entre os óleos essenciais utilizados, o diferencial de inibição de crescimento dos microrganismos testados, ocorreu na seguinte seqüência: citral com capacidade de inibição igual a P. pseudocaryophyllus var. pseudocaryophyllus procedente de Campos do Jordão, da ordem de $72 \%$, e $P$. pseudocaryophyllus var. pseudocaryophyllus procedente de Itaquaquecetuba, com inibição da ordem de $38 \%$.

Com exceção dos tratamentos com Escherichia coli, os demais microrganismos apresentaram interação significativa com o óleo essencial testado, conforme demonstrada na análise de variância, cujo desdobramento é apresentado na (Tabela 3).

A interação óleo e microrganismo apresentou os dados colocados na (Tabela 3), onde a inibição do fungo Aspergillus niger deu-se na seguinte sequência: inibição total do crescimento em citral e Pimenta pseudocaryophyllus var. pseudocaryophyllus procedente de Campos do Jordão, e reduzida inibição em óleo de $P$. pseudocaryophyllus var. pseudocaryophyllus procedente de Itaquaquecetuba, na ordem de $35 \%$.

A inibição do fungo Penicillium verrucosum deu-se na seguinte sequência: inibição total do crescimento em citral e $P$. pseudocaryophyllus de Campos do Jordão, e reduzida inibição em óleo de $P$. pseudocaryophyllus de Itaquaquecetuba, na ordem de $70 \%$.

A inibição do crescimento da bactéria Escherichia coli ocorreu na mesma proporção nos três tratamentos testados, com média de $18 \%$.

A inibição da bactéria Staphylococcus 
Tabela 2. Análise de variância e valores de $F$ e porcentagem de inibição de crescimento de microrganismos em óleo essencial de Pimenta pseudocaryophyllus var. pseudocaryophyllus e citral.

\begin{tabular}{|c|c|c|c|c|c|}
\hline $\begin{array}{l}\text { Microorganismo } \\
\text { óleo essencial }\end{array}$ & $\begin{array}{c}\text { Escherichia } \\
\text { coli } \\
\%\end{array}$ & $\begin{array}{c}\text { Penicillium } \\
\text { verrucosum } \\
\%\end{array}$ & $\begin{array}{c}\text { Staphylococcus } \\
\text { aureus } \\
\%\end{array}$ & $\begin{array}{c}\text { Aspergillus } \\
\text { niger } \\
\%\end{array}$ & $\begin{array}{c}\text { Média } \\
\%\end{array}$ \\
\hline P. pseudocaryophyllus, Campos do Jordão & 15,9159 & 90,0000 & 90,0000 & 90,0000 & 71,4790 \\
\hline P. pseudocaryophyllus, Itaquaquecetuba & 18,4579 & 70,0044 & 30,8436 & 35,7701 & 38,7690 \\
\hline Citral & 20,7221 & 90,0000 & 90,0000 & 90,0000 & 72,6805 \\
\hline Média do microrganismo & 18,3653 & 83,3348 & 70,2812 & 71,9234 & 60,9761 \\
\hline Óleo essencial & $154,1598^{* *}$ & & & & \\
\hline Fator & Valor de F & & & & \\
\hline Microrganismo & $262,5354^{* *}$ & & & & \\
\hline Interação óleo x microrganismo & $27,9302^{* *}$ & & & & \\
\hline \multicolumn{6}{|l|}{ Desdobramento de óleo em microrganismo } \\
\hline Aspergillus niger & $102,0459^{* *}$ & & & & \\
\hline Escherichia coli & $0,6018^{* *}$ & & & & \\
\hline Penicillium verrucosum & $13,8735^{* *}$ & & & & \\
\hline Staphylococcus aureus & $121,4290^{* *}$ & & & & \\
\hline \multicolumn{6}{|l|}{ Desdobramento de microrganismo em óleo } \\
\hline Citral & $124,9017^{\text {ns }}$ & & & & \\
\hline Pimenta pseudocaryophyllus - C. Jordão & $142,8333^{* *}$ & & & & \\
\hline Pimenta pseudocaryophyllus - Itaquaquecetuba & $50,6607^{* *}$ & & & & \\
\hline Média Geral & $60,9762 \%$ & & & & \\
\hline Desvio Padrão & 5,3684 & & & & \\
\hline Coeficiente de Variação & $8,8040 \%$ & & & & \\
\hline
\end{tabular}

Tabela 3. Teste de Tukey e aplicado na porcentagem de inibição de crescimento e desdobramento das interações

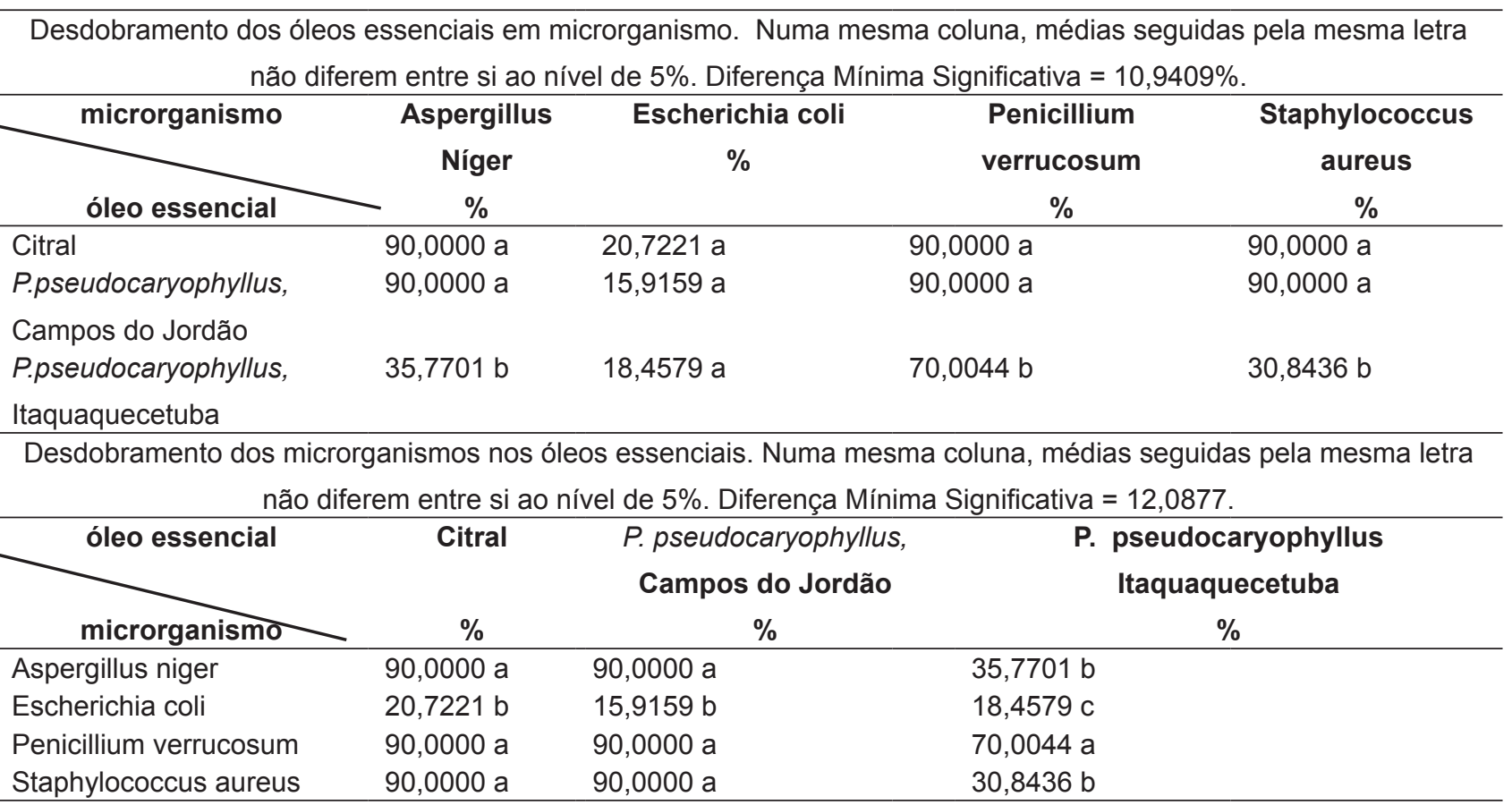

Rev. Bras. PI. Med., Campinas, v.16, n.3, p.513-520, 2014. 
aureus deu-se na seguinte seqüência: inibição total do crescimento em citral e $P$. pseudocaryophyllus de Campos do Jordão, e reduzida inibição em $P$. pseudocaryophyllus de Itaquaquecetuba, na ordem de $30 \%$.

Ainibição do crescimento dos microrganismos em óleo de P. pseudocaryophyllus de Campos do Jordão deu-se na seguinte seqüência: inibição total do crescimento de Penicillium verrucosum, da mesma forma que em Staphylococcus aureus e Aspergillus niger, e reduzida inibição de crescimento de Escherichia coli.

A inibição do crescimento dos microrganismos em óleo de P. pseudocaryophyllus de Itaquaquecetuba deu-se na seguinte seqüência: maior inibição do crescimento de Penicillium verrucosum; reduzida inibição de crescimento de Aspergillus niger, similar a Staphylococcus aureus, e maior inibição de crescimento de Escherichia coli.

Ainibição do crescimento dos microrganismos em citral deu-se na seguinte sequência: inibição total do crescimento de Penicillium verrucosum, Aspergillus niger e Staphylococcus aureus, e reduzida inibição de crescimento de Escherichia coli, na ordem de $20 \%$.

A atividade biológica do óleo essencial de P. pseudocaryophyllus coletado tanto no Parque Estadual de Campos do Jordão como em Itaquaquecetuba, apresentou variações na inibição do crescimento de acordo com os microorganismos utilizados. Porém, o óleo essencial extraído de $P$. pseudocaryophyllus procedente de Campos do Jordão apresentou atividade biológica superior à espécie coletada em Itaquaquecetuba.

Estes resultados demonstram que o óleo essencial de P. pseudocaryophyllus var pseudocaryophyllus, tem potencial de aproveitamento como agente antimicrobiano, havendo necessidade de efetuar estudos quanto à biologia da espécie, a influência do ambiente e procedências, e a determinação da atividade biológica de todos os componentes químicos do óleo.

\section{CONCLUSÕES}

A atividade biológica do óleo essencial de P. pseudocaryophyllus var. pseudocaryophyllus coletado tanto no Parque Estadual de Campos do Jordão como em Itaquaquecetuba, apresentou variações na inibição do crescimento de acordo com os microorganismos utilizados. Porém, o óleo essencial extraído de P. pseudocaryophyllus procedente de Campos do Jordão apresentou atividade biológica superior à espécie coletada em Itaquaquecetuba. O óleo essencial das folhas $P$. pseudocaryophyllus mostrou uma boa atividade contra os microrganismos testados, mostrando tratar-se de uma espécie com potencial para uso nas indústrias químico-farmaceutica, no desenvolvimento de fármacos, uma vez que a espécie contém componentes químicos de conhecida atividade biológica no seu óleo essencial.

$O$ rendimento do óleo essencial de $P$. pseudocaryophyllus procedente de Campos do Jordão $(2,3 \%)$, foi superior ao de Itaquaquecetuba $(1,9 \%)$.

Este elevado rendimento em óleos essenciais torna a Pimenta pseudocaryophyllus var. pseudocaryophyllus uma espécie interessante e promissora.

\section{REFERÊNCIA}

APEL, M.A. et al. Investigação da atividade antimicrobiana do óleo volátil de espécies da família Myrtaceae. In: SIMPÓSIOS DE PLANTAS MEDICINAIS DO BRASIL, 15, 1998, Água de Lindóia. Resumos... São Paulo: \{S.I.:s.n.\}, 1998, p. 51.

AURORE, G.S. et al. Antibacterial and antifungal activities of the essential oils of Pimenta racemosa var. racemosa P.Miller (J.W.Moore) Myrtaceae. Journal Essential Oil Research, Carol Stream, v. 10, n. 2, p.161-164, 1998.

BARATA, L.E.S.; VILA, A.M.; CARVALHO, R.Q. Mercado de perfumaria e cosmética no Brasil. In: SIMPÓSIO BRASILEIRO DE ÓLEOS ESSENCIAIS, 3, 2005, Campinas. Documentos IAC. Campinas: Instituto Agronômico de Campinas, (comunicação oral). 2005

BIZZO, H.R., HOVELL, A.M. C., REZENDE, C.M. Óleos Essenciais no Brasil: Aspectos Gerais, Desenvolvimento e Perspectivas. Química Nova, Editora: Sociedade Brasileira de Química (SBQ) / Centro de Gestão e Estudos Estratégicos (CGEE) ISSN: 0100-4042 v. 32. $\mathrm{n}^{\circ} 3$, p. 588-594. 2009.

BARROSO, G.M. Myrtaceae. In: Sistemática de angiosperma do Brasil. Viçosa: Universitária Federal de Viçosa: Imprensa Universitária, v. II. 1984. 377p.

CAMPOS CORRÊA, R.G. et al. Óleos essenciais de espécies do gênero Pseudocaryophyllus In: CONGRESSO INTERNACIONAL DE ÓLEOS ESSENCIAIS, 5. Anais... Rio de Janeiro, 1972, p. 307-309.

CAMPOS CORREA, R.G., GOTTLIEB, O.R Óleos essenciais de espécies do gênero Pseudocaryophyllus. Anais da Academia Brasileira de Ciências, v. 44, p. 307-309, 1970.

CHALCHAT, J.C. et al. Correlation between chemical composition and antimicrobial activity. VI. Activity of some African essential oils. Journal Essential Oil Research, Carol Stream, v. 9, n. 1, p. 67-75, 1997.

CRAVEIRO, A.A.; MACHADO, M.I.L. De aromas, insetos e plantas. Ciência Hoje, v. 4, n. 23, p. 54-63, 1986.

CUSTÓDIO, D.L. et al. Estudo do óleo essencial de Pimenta pseudocaryophyllus (Gomes) L.R. Landrum. In: SIMPÓSIO BRASILEIRO DE ÓLEOS ESSENCIAIS, 4, 2007, Fortaleza. Resumos... Ceará: PADETEC- Parque de Desenvolvimento Tecnológico, 2007. p. 22.

DE ALENCAR, R. et al. Óleos essenciais de plantas 
brasileiras. Acta Amazônica, v. 1, n. 3, p. 41-43, 1971.

EDINGTON, L.V.K.L.; KHEW, G.L. Fungitoxic spectrum benzimidazole compounds. Phytopathology, v. 59, p. $718-724,1971$.

FENIK, I.J.S.; RETAMAR, J.A. El aceite esencial de Pseudocaryophyllus guilli (Guili). In : CONGRESSO INTERNACIONAL DE ÓLEOS ESSENCIAIS, 5, Anais da Academia Brasileira de Ciências, Rio de Janeiro, 1972, p. 175-180.

GAUTHIER, R.; AGOUMI, A.; GOURAI, M. Activité déxtrats de Myrtus communis contre Pediculus Humanus Capitis. Plantes médicinales et phytotherapie. Journal de Pharmacognosie, v. 23, n. 2, p. 95-108, 1989.

GOTTLIEB, O.R.; BORIN, M.R. de M.B. Diversidade da flora odorífera brasileira. In: SIMPÓSIO BRASILEIRO DE ÓLEOS ESSENCIAIS - DIAGNÓSTICO \& PERSPECTIVA, 2, 2003. Resumos... Campinas: Instituto Agronômico de Campinas, 2003. p. 1.

HOEHNE, F.C. Plantas e substâncias vegetais tóxicas e medicinais. São Paulo: Novos Horizontes Ed. Ltda., 1939, 355 p.

JARDIM. A.S.; JACCOUD, R.J.S. Estudo farmacognóstico da "erva doce do mato". Revista Brasileira Farmácia, v. 46, n.3. p.3-17, 1965.

LANDRUM, L.R.; KAWAZAKI, M.L. The genera of Myrtaceae in Brazil: an illustrated synoptic treatment and identification Keys. Brittonia, n.4, p. 508-36, 1997.

LIMBERGER, R.P. et al. Óleo volátil de Marlierea eugeniopsoides e Marlierea obscura (Myrtaceae) composição química e atividade antimicrobiana. In: SIMPÓSIO DE PLANTAS MEDICINAIS DO BRASIL, 15, 1998, Água de Lindóia. Resumos... \{S.I.:S.n.\}, 1998. p. 158.

LIMA, M.E.L. et al. Antimicrobial activity of the essential oil from two specimens of Pimenta pseudocaryophyllus
(GOMES) L.R. Landrum (Myrtaceae) native from São Paulo State - Brazil. Pharmacology on line, California State Library, v. 3, p. 589-593, 2006.

NAKAOKA SAKITA, M. et al. Óleo essencial de Pimenta pseudocaryophyllus var. pseudocaryophyllus (Gomes) Landrum (Myrtaceae) I: Cromatografia a gás/ espectrometria de massa (CG/EM). Revista Instituto Florestal, v. 6, n. único, p. 53- 61, 1994.

PAULA, J.A.M. et. al. Gênero Pimenta: aspectos botânicos, composição química e potencial farmacológico. Revista Brasileira de Plantas Medicinais. v.12,n.3, p.363-379, 2010.

PAULA, C.J.R. et al. Estudo da composição química do óleo essencial de Eugenia dysenterica D. In: SIMPÓSIO DE PLANTAS MEDICINAIS DO BRASIL, 15, 1998, Água de Lindóia. Resumos... São Paulo: \{S.I.:s.n.\}, 1998. p.156.

PAULA, J.A.M. et al. Antimicrobial activity of the crude ethanol extract from Pimenta pseudocaryophyllus. Pharmaceutical Biology, v.47, n.10, p.987-93, 2009.

PEREIRA, R.S. et al. Atividade antibacteriana de óleos essenciais em cepas isoladas de infecção urinária. Revista Saúde Pública, v. 38, n. 2, p. 1-5, 2004.

PLANT Pathologist's Pocketbook. Kew Surrey: The Commonwealth Mycological Institute, 1968. 236 p.

ROSS, J.L.S.; MOROZ, I.C. Mapa geomorfológico do Estado de São Paulo. IPT/ FAPESP, 1997, 63 p.

VIANE, M.C.E. et al. Atividade antimicrobiana e composição química do óleo essencial de Eugenia speciosa CAMB. (Myrtaceae). In: SIMPÓSIO DE PLANTAS MEDICINAIS DO BRASIL, 15, 1998, Água de Lindóia. Resumos... \{S.I.:S.n.\}, 1998, p. 45.

WASICKY, R. Uma modificação do aparelho de Clevenger para extração de óleos essenciais. Revista da Faculdade Farmácia e Bioquímica, v. 1 n.1, p. 77 - 81. 1963. 